\title{
Heat-Induced Epithelial Barrier Dysfunction Occurs via C-Src Kinase and P120ctn Expression Regulation in the Lungs
}

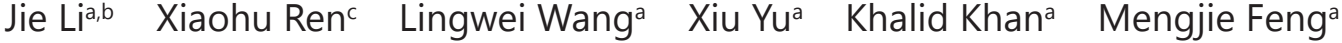 \\ Qi Hea Huanmin Luo ${ }^{d}$ Chen Qiu
}

\begin{abstract}
aDepartment of Respiratory Diseases, Second Clinical Medical College (Shenzhen People's Hospital), Jinan University, Shenzhen, 'Integrated Chinese and Western Medicine Postdoctoral Research Station, Jinan University, Guangzhou, 'Key Laboratory of Modern Toxicology of Shenzhen, Shenzhen Center for Disease Control and Prevention, Shenzhen, dDepartment of Pharmacology, School of Medicine, Jinan University, Guangzhou, China
\end{abstract}

\section{Key Words}

Heat $•$ Thermal inhalation injury $\bullet P 120 c t n \cdot C-S r c \cdot C e l l$ junction

\begin{abstract}
Background/Aims: Thermal injury causes pulmonary edema and can lead to death. Intercellular junctions are composed of adhesive (p120ctn, E-cadherin, $\alpha$-catenin and $\beta$-catenin) and compact (occludin and ZO-1) junctions. Heat deteriorates intercellular junctions and increases cell gaps to ultimately induce pulmonary edema, but the underlying mechanism remains elusive. Methods: Mouse lung epithelial (MLE-12) cells pre-treated with the c-Src inhibitor PP2, p120ctn catenin (p120ctn) small interfering RNA and p120ctn catenin (p120ctn) complementary DNA were subjected to heat treatment. Western blotting and realtime polymerase chain reaction assays were used to evaluate junction protein expression changes after heat treatment, and co-immunoprecipitation was used to test the binding state of junction proteins. In addition, hematoxylin and eosin staining and immunohistochemistry were used to evaluate changes in junction protein expression and lung injury in a Wistar rat model of thermal inhalation injury. Results: Heat increased cell permeability; induced ZO1 , occludin, $\alpha$-catenin and $\beta$-catenin degradation; and decreased E-cadherin distribution in cell membranes. Heat also activated c-Src and decreased both p120ctn expression levels and occludin and ZO-1 association. c-Src inhibitor (PP2) treatment and p120ctn overexpression reversed these effects and attenuated lung injury in vivo. Conclusion: Heat induces junction protein degradation and dissociation to increase membrane permeability and cause lung edema via c-Src kinase activation and p120ctn expression downregulation.

\section{Introduction}

Heat is a major risk factor for lung damage in thermal inhalation injury, which is common in burn patients and can induce acute lung injury [1]. Thermal injury causes pulmonary

Chen Qiu

and Huanmin Luo

KARGER
Dept. of Respiratory Diseases, Second Clinical Medical College (Shenzhen People's Hospital), Jinan Univ. 1017 Dongmen North Road, Luohu District, Shenzhen,

Guangdong, 518020, (China); E-Mail qiuchen@email.jnu.edu.cn / tlhm@jnu.edu.cn 


\section{Cellular Physiology Cell Physiol Biochem 2018;48:237-250 \\ \begin{tabular}{l|l} 
and Biochemistry $10.1159 / 000491723$ & $\begin{array}{l}\text { D } 2018 \text { The Author(s). Published by S. Karger AG, Base } \\
\text { www.karger.com/cpb }\end{array}$ \\
\cline { 2 - 3 }
\end{tabular} \\ Li et al.: Epithelial Barrier Dysfunction Occurs via C-Src Kinase and P120ctn Expression Regulation}

edema, which can lead to acute respiratory distress syndrome, sepsis, multiorgan failure and even death [2]. Alveolar epithelial cells are the main barrier protecting the lungs from endogenous and exogenous damage. Heat causes cell junction deterioration and increases gaps between these cells, ultimately inducing pulmonary edema, which is the leading cause of mortality in thermal inhalation injury [3]. However, the mechanism through which heat destroys cell connections and leads to pulmonary dysfunction remains elusive. Treatments that maintain alveolar membrane integrity and pulmonary vascular permeability after heat exposure are crucial for patients with thermal inhalation injury [4]. Therefore, a detailed understanding of cell junction protein structure and function is highly significant for preventing and treating heat-induced lung injury.

Pulmonary edema and lung injury occur through a series of reactions and inflammatory responses induced by heat $[5,6]$. Alveolar barrier function loss is a hallmark of severe lung injury because the junctions between endothelial and epithelial cells regulate paracellular fluid flux. Heat also reportedly induces intestinal epithelial barrier dysfunction [7]. The destabilization of cell-cell contacts involved in maintaining endothelial barrier function can lead to increased endothelial permeability. This increase in endothelial permeability results in the anarchical movement of fluid, solutes and cells out of the vasculature and into the surrounding tissues, thereby contributing to various diseases, such as stroke or pulmonary edema $[8,9]$. The results of studies performed during the past three decades have shown that hyperthermia changes junction protein expression and increases paracellular permeability in in vitro culture models. The most direct indicator of permeability is cell connectivity, which is mediated by adherens junctions and tight junctions and plays an indispensable and key role in maintaining the integrity of the epithelial barrier. The main components of adherens junctions include p120ctn, E-cadherin, $\alpha$-catenin and $\beta$-catenin, whereas compact junctions are composed primarily of occludin and ZO-1 [10]. In epithelial cells, external stimuli, such as inflammation and hypoxia, can activate intracellular tyrosine kinases (tyrosine pressure protein kinase or c-Src) and trigger a series of inflammatory responses [11]. c-Src is a member of the Src family, which is involved in intracellular signal transduction [12]. Studies have shown that PL20 is a c-Src kinase substrate that contributes to the degradation and synthesis of tight junction proteins. c-Src kinase activation can initiate p120ctn degradation to disrupt alveolar epithelial barrier integrity in ventilator-induced lung injury [12]. However, the functions of c-Src and p120ctn in thermal inhalation injury require further research.

In the present study, we hypothesized that heat degrades membrane integrity and increases pulmonary vascular permeability in thermal inhalation injury. Specifically, heat induces c-Src activation to cause p120ctn degradation, which disturbs cell protein junctions and reduces the association of occludin and ZO-1, thus causing cause alveolar epithelial barrier dysfunction and pulmonary hyperpermeability.

\section{Materials and Methods}

\section{Cell culture and treatment}

MLE-12 cells (Cell Resource Center, Beijing, China) were cultured at $37^{\circ} \mathrm{C}$ in a $5 \% \mathrm{CO}_{2}$ humidifiedatmosphere in DMEM (Dulbecco's modified Eagle's medium) (Gibco, CA, USA) containing 10\% fetal bovine serum and $1 \%$ penicillin-streptomycin. The cells were incubated at $52^{\circ} \mathrm{C}$ for $5 \mathrm{~min}$ for the in vitro heat exposure model. PP2 and SU66656 (Thermo Scientific, Fremont, CA, USA) were dissolved in dimethyl sulfoxide (DMSO) and freshly diluted for each experiment. The DMSO concentration in the culture medium was less than $0.1 \%$ in all the experiments. The cells were treated with the PP2 inhibitor $(10 \mu \mathrm{m})$ and SU66656 $(50 \mathrm{~nm}) 4 \mathrm{~h}$ before the heat treatment. MLE-12 cells were incubated at $47^{\circ} \mathrm{C}$ for $5 \mathrm{~min}$ to produce a heat exposure damage model. To investigate the changes in lung epithelial cell integrity after heat treatment, we used FITC-dextran and TER to measure the lung epithelial cell permeability.

Animals and thermal inhalation injury

The animal studies were approved by the Animal Ethics Committee of the Third Military Medical University and were conducted according to the approved guidelines. Thermal inhalation injury was induced 


\section{Cellular Physiology Cell Physiol Biochem 2018;48:237-250 \\ \begin{tabular}{ll|l} 
DOI: 10.1159/000491723 & $\begin{array}{l}\text { () } 2018 \text { The Author(s). Published by S. Karger AG, Base } \\
\text { www.karger.com/cpb }\end{array}$ \\
\cline { 2 - 3 }
\end{tabular} \\ Li et al.: Epithelial Barrier Dysfunction Occurs via C-Src Kinase and P120ctn Expression Regulation}

as previously described [13]; adult male Sprague-Dawley rats were used for the experiments. All procedures were approved by the Animal Ethics Committee of Jinan University and were carried out in accordance with the approved guidelines. The thermal inhalation injury procedure was modified from a previously described method used in dogs [13]. Adult male Sprague-Dawley rats were placed under general anesthesia by constant inhalation of isoflurane (5\%) via a face mask. Tracheotomies were performed under anesthesia, and a 1-cm catheter was inserted into the trachea; preheated dry air was blown through the catheter into the airway by a heat gun (TAK-3316E, Shenzhen Takgiko Technology, Shenzhen, China) at a flow rate of $190 \mathrm{~L} / \mathrm{min}$ for a total of $10 \mathrm{~min}$ with $1 \mathrm{~min}$ intervals in between each minute of heat inhalation. The air was preheated to $110^{\circ} \mathrm{C}$ inside the heat gun and was determined to be approximately $50^{\circ} \mathrm{C}$ when blown into the end of the catheter. Then, the rats were closed, and the wounds were sutured; acetylpromazine $(0.75 \mathrm{mg} / \mathrm{kg}$ body weight) was injected intraperitoneally to relieve pain.

\section{Western blotting}

RIPA lysis buffer was used to extract protein from cells and lung tissue (80-120 mg). After protein concentration determination, equal amounts of protein were subjected to 6-8\% polyacrylamide-sodium dodecyl sulfate gel electrophoresis and transferred as previously described. Membrane strips were blocked with $5 \%$ non-fat milk for $1 \mathrm{~h}$ at room temperature and incubated with primary antibodies overnight at $4^{\circ} \mathrm{C}$. The following antibodies were used for detection: E-cadherin, occludin (Santa Cruz Biotechnology, Santa Cruz, CA, USA), $\beta$-catenin and $\alpha$-catenin (Life Technologies, Germany). $\beta$-actin or $\beta$-tubulin (Santa Cruz Biotechnology) was detected on the same membrane as the control to ensure equal protein loading. After incubation with secondary horseradish peroxidase-labeled antibodies (Abcam, UK; diluted 1:5000 in blocking buffer), the membranes were washed three times, incubated with an enhanced chemiluminescence substrate developing solution and detected with a FluorChem E System (Alpha Innotech, San Leandro, CA, USA). Densitometry was performed using ImageJ (National Institutes of Health, Bethesda, MD, USA). All experiments were performed in triplicate.

\section{Immunoprecipitation}

Protein was extracted on ice for 30 min using RIPA lysis buffer; the samples were ultrasonicated and centrifuged at $16,000 \times \mathrm{g}$ for $5 \mathrm{~min}$ at $4^{\circ} \mathrm{C}$. After protein concentration determination, the lysates were mixed with mouse immunoglobulin (Ig) $\mathrm{G}$ and agarose $\mathrm{A} / \mathrm{G}$ on a shaker for $5 \mathrm{~h}$ at $4^{\circ} \mathrm{C}$. Then, the samples were centrifuged at 13, $000 \times \mathrm{g}$ for $5 \mathrm{~min}$. After the supernatants were collected, a primary antibody was added to pull down the protein complexes, and the mixtures were incubated overnight at $44^{\circ} \mathrm{C}$ on a shaker. The agarose was washed five times with centrifugation at $1300 \mathrm{rpm}$ and then dissolved in sodium dodecyl sulfate gel sample buffer. The samples were analyzed using the western blotting protocol.

\section{p120ctn small interfering RNA (siRNA) and p120ctn complementary DNA (cDNA) transfection}

Mouse lung epithelial (MLE-12) cells (70\% confluent) were cultured in optim media without serum for $2 \mathrm{~h}$. Then, the scrambled siRNA and p120ctn siRNA (Santa Cruz Biotechnology) were transfected using Lipofectamine ${ }^{\circledR}$ RNAiMAX transfection reagent (Invitrogen, MA, USA) according to the manufacturer's protocol [7]. The p120ctn 1A cDNA and the empty vector (GeneChem Inc., Shanghai, China) were transfected using Lipofectamine 2000 cDNA reagent (Invitrogen, MA, USA) according to the manufacturer's protocol. After $12 \mathrm{~h}$, the cell culture media was exchanged for fresh complete media. Forty-eight hours after transfection, the cells were used for the experiments.

To overexpress p120ctn in vivo, we injected liposome-based vectors into the retinal vein plexus for the systemic and targeted delivery of the p120ctn cDNA complex. After p120ctn siRNA and p120ctn cDNA transfection, we treated lung epithelial cells with heat at $47^{\circ} \mathrm{C}$ for $5 \mathrm{~min}$ and then returned them to normal cell culture conditions. After 2 days, we measured E-cadherin expression levels in the cell membrane and cytoplasm. Western blotting was used to detect the transfection efficiency 2 days later; at this point, we established the inhalation injury model. In some experiments, the animals were intravenously administered the c-Src inhibitor PP2 $(2 \mu \mathrm{g} / \mathrm{kg})$ dissolved in dimethyl sulfoxide $1 \mathrm{~h}$ before inhalation injury.

Quantitative real-time reverse transcription-polymerase chain reaction (PCR)

Total RNA was extracted from $30 \mu \mathrm{g}$ of cell protein and $100 \mathrm{mg}$ of lung tissue using Trizol reagent. After the total RNA concentration was measured, $1 \mu \mathrm{g}$ of total RNA was used for reverse transcription. 


\section{Cellular Physiology Cell Physiol Biochem 2018;48:237-250 and Biochemistry DOI: 10.1159/000491723 1 (O) 2018 The Author(s). Published by S. Karger AG, Basel and Biochemistry

Subsequently, quantitative real-time reverse transcription-PCR was carried out using a cDNA synthesis kit (Thermo Scientific, Fremont, CA, USA) and the SGExcelUltraSYBR Mixture (Sangon Biotech, Shanghai, China).

\section{Measurement of transepithelial electrical resistance (TER)}

MLE-12 cells were seeded at $2.0 \times 10^{6}$ cells per well on polycarbonate membranes in Transwell inserts (6.5-mm diameter, $0.4-\mu \mathrm{m}$ pore size; Corning, Cambridge, MA, USA). Data were collected when the TER values were consistently above $300 ' \Omega / \mathrm{cm}^{2}$. The TER values for the filter-grown MLE-12 intestinal monolayers were measured with a Millicell ERS (electrical resistance system; Millipore, Bedford, MA, USA). All TER values are expressed as $\Omega / \mathrm{cm}^{2}$.

\section{Epithelial paracellular permeability assay}

MLE-12 paracellular permeability across cell monolayers was measured using the flux of fluorescein isothiocyanate (FITC)-labeled dextran with a molecular mass of $4 \mathrm{kDa}$ (FD4, Sigma-Aldrich). FD4 (1 mg/ $\mathrm{mL}$ ) was added to the medium in the apical chambers of Transwells (Corning). After $2 \mathrm{~h}$, the medium was collected from the bottom chamber, and the fluorescence was measured with a fluorometer (excitation, 492 $\mathrm{nm}$; emission, $520 \mathrm{~nm}$; BioTek).

\section{Histopathologic analysis}

Lung samples (sham, Heat, DMSO, PP2 and P120cDNA) were isolated and fixed in 4\% paraformaldehyde $(4 \mathrm{~h})$ and embedded in paraffin. Paraffin sections were stained with hematoxylin and eosin as previously described [14]. The slides were observed using a fluorescence microscope (Instruments Inc., USA) and quantified by randomly counting 30 areas from three independent experiments. The degree of lung pathological injury was scored based on the following variables: inflammatory cell infiltration, lung edema, hemorrhaging, hyaline membrane structure and atelectasis. The degree of each abnormality was graded numerically from 0 (normal) to 4 (diffuse injury) according to the following criteria (19): no injury $=0$; injury to $25 \%$ of the field $=1$; injury to $50 \%$ of the field $=2$; injury to $75 \%$ of the field $=3$; diffuse injury $=4$. Blind analyses were performed to determine the lesion degree of all samples [15].

\section{Immunohistochemical staining}

Immunohistochemistry analyses were performed using paraffin-embedded lung sections (2-4 mm). The sections were deparaffinized in xylene and dehydrated in a gradient of ethanol dilutions. A proteolytic enzyme solution was used for antigen retrieval. Then, the sections were incubated with rabbit IgG, antioccludin (Santa Cruz Biotechnology) and anti-ZO-1 (Invitrogen, Paisley, UK) antibodies with 5\% non-fat milk overnight. On the second day, the slides were washed three times and incubated with biotinylated anti-rabbit IgG goat IgG (Sigma-Aldrich, Shanghai, China) and then with an ABC-alkaline phosphatase kit (Vector, Burlingame, CA, USA). Images were taken with a Leica Microsystems apparatus (DFC300FX, Leica, Switzerland) and quantified with Image Pro-Plus. Five random fields were examined per animal to detect ZO-1 and occludin expression levels [16]. ZO-1 and occluding expression levels were also quantified using Image J software. Briefly, after the figures were transformed into 8-bit images, immunohistochemistry images were acquired using identical parameters, and the background intensity was subtracted. Then, the ZO-1 staining intensity was measured. The reported values are the averages of different images from five rats [17].

\section{Determination of the wet-to-dry weight $(W / D)$ ratio}

Wet weights were obtained immediately after fresh lung tissues were isolated. Then, $100 \mathrm{mg}$ of lung tissue was dried in an oven at $60^{\circ} \mathrm{C}$ for 2 days; at this point, the stable weight was recorded as the dry weight. Then, the W/D ratio was calculated.

\section{Statistical analysis}

Statistical analyses were performed by using t-tests and one-way ANOVA. The data are presented as the means \pm standard error of three independent experiments for each group and were analyzed with GraphPad Prism 6 (GraphPad Software, Inc., La Jolla, CA, USA). All tests were two-tailed. A P value of $<0.05$ was considered significant. 


\section{Cellular Physiology \\ Cell Physiol Biochem 2018;48:237-250 and Biochemistry \\ Li et al.: Epithelial Barrier Dysfunction Occurs via C-Src Kinase and P120ctn Expression Regulation}

\section{Results}

Decreased junction protein expression levels and increased paracellular permeability in lung epithelial cells after heat treatment

As shown in Fig. 1A, no changes in these parameters were observed at time points $0 \mathrm{~h}$ and $12 \mathrm{~h}$ after heat treatment. However, the paracellular permeability was increased on days 1 and 3. To further evaluate the damage to junction proteins caused by heat exposure, we assessed the expression levels of ZO-1, occludin, $\beta$-catenin and $\alpha$-catenin in lung epithelial cells after heat treatment. The real-time PCR results indicated that the levels of these factors were decreased on days 1 and 3 after heat treatment (Fig. 1B). Consistently, the protein expression levels were also decreased on days 1 and 3 (Fig. 1C). Furthermore, we isolated cell membrane and cytoplasmic proteins to examine the effect of heat on E-cadherin distribution. On days 1 and 3 , the E-cadherin levels in the cell membrane were decreased significantly, whereas the levels in the cytoplasm were increased compared with those in the control group (Fig. 1D).

Decrease in junction protein binding in heat-treated lung epithelial cells

Junction protein binding states are critical for maintaining cell permeability. To further explore the mechanism through which heat affects junction proteins, we analyzed the binding states of junction proteins with coimmunoprecipitation. As shown in Fig. 2A, the association of PL20 and E-cadherin was decreased on days 1 and 3 after heat treatment. Similarly, occludin and ZO-1 protein binding was reduced. These results indicate that the heat treatment negatively affected the junction binding states of the lung epithelial cells (Fig. 2B).

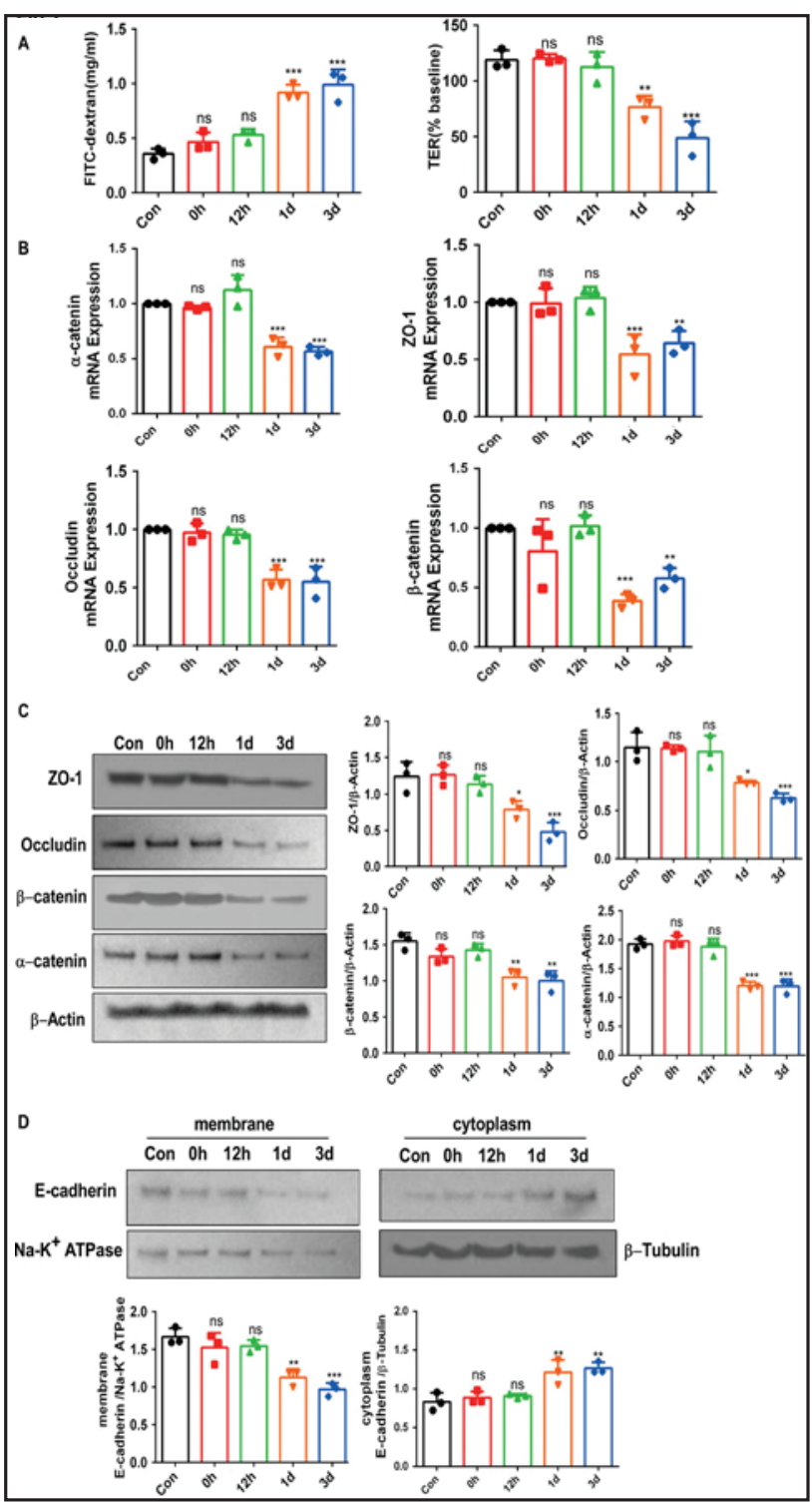

Fig. 1. Heat treatment increased permeability and decreased junction protein expression levels in lung epithelial cells. (A) Paracellular permeability was analyzed with fluorescein isothiocyanate-dextran and transepithelial electrical resistance. ns, no significant difference, ${ }^{* *} \mathrm{P}<0.01$ and ${ }^{* * *} \mathrm{P}<0.001$ vs. control $(\mathrm{n}=3)$. (B) Real-time polymerase chain reaction analysis and quantification of the expression of the cell junction proteins ZO- 1 , occludin, $\beta$-catenin and $\alpha$-catenin. Relative messenger RNA (mRNA) levels were normalized to the $18 \mathrm{~s}$ ribosomal RNA levels. ns, no significant difference, ${ }^{* *} \mathrm{P}<0.01$ and ${ }^{* * *} \mathrm{P}<0.0001$ vs. control $(\mathrm{n} \geq 3)$. (C) Representative western blot and quantification data for the cell junction proteins ZO-1, occludin, p120ctn, $\beta$-catenin and $\alpha$-catenin. $\mathrm{P}^{*}<0.05,{ }^{* *} \mathrm{P}<0.01$ and ${ }^{* * *} \mathrm{P}<0.0001$ vs. control. (D) Representative western blot and quantification data for E-cadherin in the cell membrane and cytoplasm. ns, no significant difference, ${ }^{*} \mathrm{P}<0.05,{ }^{* *} \mathrm{P}<0.01$ and ${ }^{* * *} \mathrm{P}<0.0001$ vs. control. 


\section{Cellular Physiology Cell Physiol Biochem 2018;48:237-250 \begin{tabular}{ll|l} 
aOI: 10.1159/000491723 & $\begin{array}{l}\text { () } 2018 \text { The Author(s). Published by S. Karger AG, Base } \\
\text { www.karger.com/cpb }\end{array}$ \\
\hline
\end{tabular} \\ Li et al.: Epithelial Barrier Dysfunction Occurs via C-Src Kinase and P120ctn Expression Regulation}

\section{Involvement of $120 c t n$ and $c$-Src in heat-induced junction protein degradation}

Heat treatment degraded p120ctn and increased phosphorylated (p)-c-Src expression levels, which means that heat treatment activated Src activity (Fig. 3A). To further elucidate the role of p120ctn and c-Src in heat-induced junction damage, we used p120ctn siRNA, p120ctn cDNA, PP2 (a c-Src inhibitor) and SU6656 to regulate the expression of p120ctn and c-Src. Under normal conditions, P120ctn cDNA and P120ctn siRNA respectively increased and decreased P120ctn expression levels (Fig. 3B). In addition, the P120ctn cDNA group expressed higher levels of P120 than the control group after the heat treatment (Fig. 3C). As we expected, both PP2 and SU6656 decreased (p)-c-Src expression, which was activated by the heat treatment (Fig. 3D and Fig. 3E). Notably, in the normal cells, we found that c-Src kinase activity was higher in the p120ctn siRNA treatment group than in the control group (Fig. 3F). Furthermore, P120ctn cDNA inhibited (p)-c-Src activation in the heat damage model, which indicated that p120ctn regulates c-Sr activity (Fig. 3G).

Heat can lead to the degradation of junction proteins (ZO-1, occludin, $\beta$-catenin and $\alpha$-catenin). However, compared with the heat treatment group, the PP2 and p120ctn cDNA treatment groups showed dramatically lower cell junction degradation. To avoid pharmacological agent off-target effects, we also used SU6656 as a c-Src inhibitor. We found that SU6656 can dramatically inhibit cell junction degradation. These results demonstrate that $\mathrm{p} 120 \mathrm{ctn} / \mathrm{c}-\mathrm{Src}$ regulated the junction protein changes after heat treatment, and p120ctn was upstream of c-Src in the regulation of c-Src activity (Fig. 3I).

\section{Effect of p120ctn on E-cadherin distribution and junction protein binding in vitro}

Under normal conditions, p120ctn siRNA transfection significantly decreased E-cadherin expression levels in the cell membrane. However, the expression levels in the cytoplasm were increased compared with those in the scrambled siRNA group (Fig. 4A). After heat treatment, E-cadherin expression levels in the cell membrane and cytoplasm decreased and increased, respectively. Notably, p120ctn cDNA treatment reversed the distribution of E-cadherin (Fig. 4B). All of these results indicate that the loss of P120ctn can decrease E-cadherin expression in the cell membrane, and heat can decrease P120ctn to cause E-cadherin departure from the membrane. These findings are compatible with the results that heat can decrease P120ctn expression (Fig. 3A) and cause E-cadherin transfer from the membrane to the cytoplasm (Fig. 1D).

We also performed coimmunoprecipitation analyses to examine junction protein binding after p120ctn cDNA and siRNA transfection. As shown in Fig. 4C and Fig. 4D, the degradation of occludin was significantly increased in the P120CTN siRNA transfection group, and the results of the coimmunoprecipitation analyses suggested that p120ctn siRNA transfection further reduced the association of $\mathrm{ZO}-1$ and occludin. Conversely, the overexpression of p120ctn reduced the degradation of occludin and increased the association of $\mathrm{ZO}-1$ and occludin.

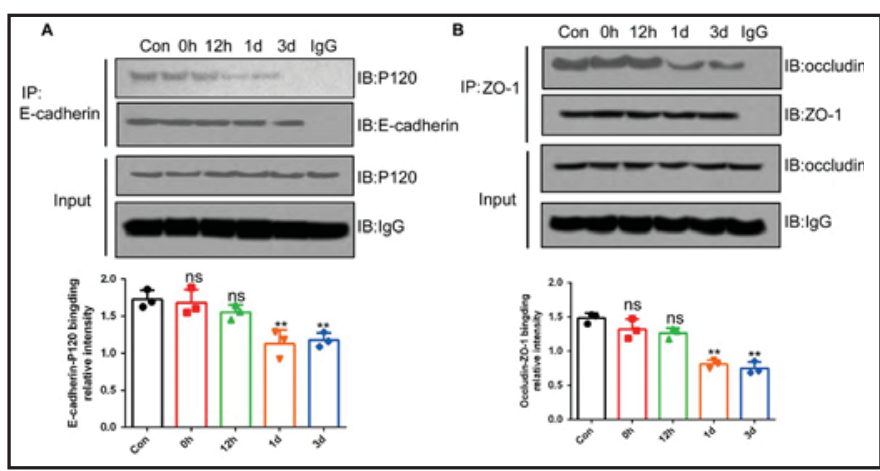

Fig. 2. Heat decreased the junction protein binding state of lung epithelial cells. (A) Co-immunoprecipitation (Co-IP) analyses were used to determine the binding of p120ctn and E-cadherin. Representative western blot and quantification data for p120ctn and E-cadherin. $\mathrm{P}^{*}<0.05$ and ${ }^{* * *} \mathrm{P}<0.001$ vs. control ( $\mathrm{n} \geq 3$ ). (B) Co-IP was used to analyze the binding of occludin and ZO-1. Representative western blot and quantification data for occludin and $\mathrm{ZO}-1 .{ }^{*} \mathrm{P}<0.05,{ }^{* * *} \mathrm{P}<0.001$ vs. control $(\mathrm{n} \geq 3)$. 


\section{Cellular Physiology Cell Physiol Biochem 2018;48:237-250 \begin{tabular}{c|c|c|} 
DOI: 10.1159/000491723 & O 2018 The Author(s). Published by S. Karger AG, Basel \\
and Biochemistry Published online: July 13, 2018 &
\end{tabular} \\ Li et al.: Epithelial Barrier Dysfunction Occurs via C-Src Kinase and P120ctn Expression Regulation}

Fig. 3. Heat decreased p120ctn expression levels and activated c-Src phosphorylation.

(A) Representative western blot and quantification data for p120ctn, phosphorylated $\mathrm{c}-\mathrm{Src}$ (p-c-Src), c-Src and $\beta$-actin at various time points after heat treatment. ns, no significant difference, $* * * \mathrm{P}<0.0001$ vs. control. (B) Representative western blots of p120ctn after p120ctn small interfering RNA (siRNA) or p120ctn complementary DNA (cDNA) treatment in normal cells. (C) Representative western blots of p120ctn after p120ctn complementary DNA (cDNA) transfection in heat-treated cells. (D) Representative western blots of p-c-Src and c-Src after PP2 treatment in heat-treated cells. (E) Representative western blots of p-c-Src and c-Src after SU6656 treatment in heat-treated cells. (F) Representative western blots of $\mathrm{p}$-c-Src and c-Src after p120ctn small interfering RNA (siRNA) treatment in normal cells. (G) Representative

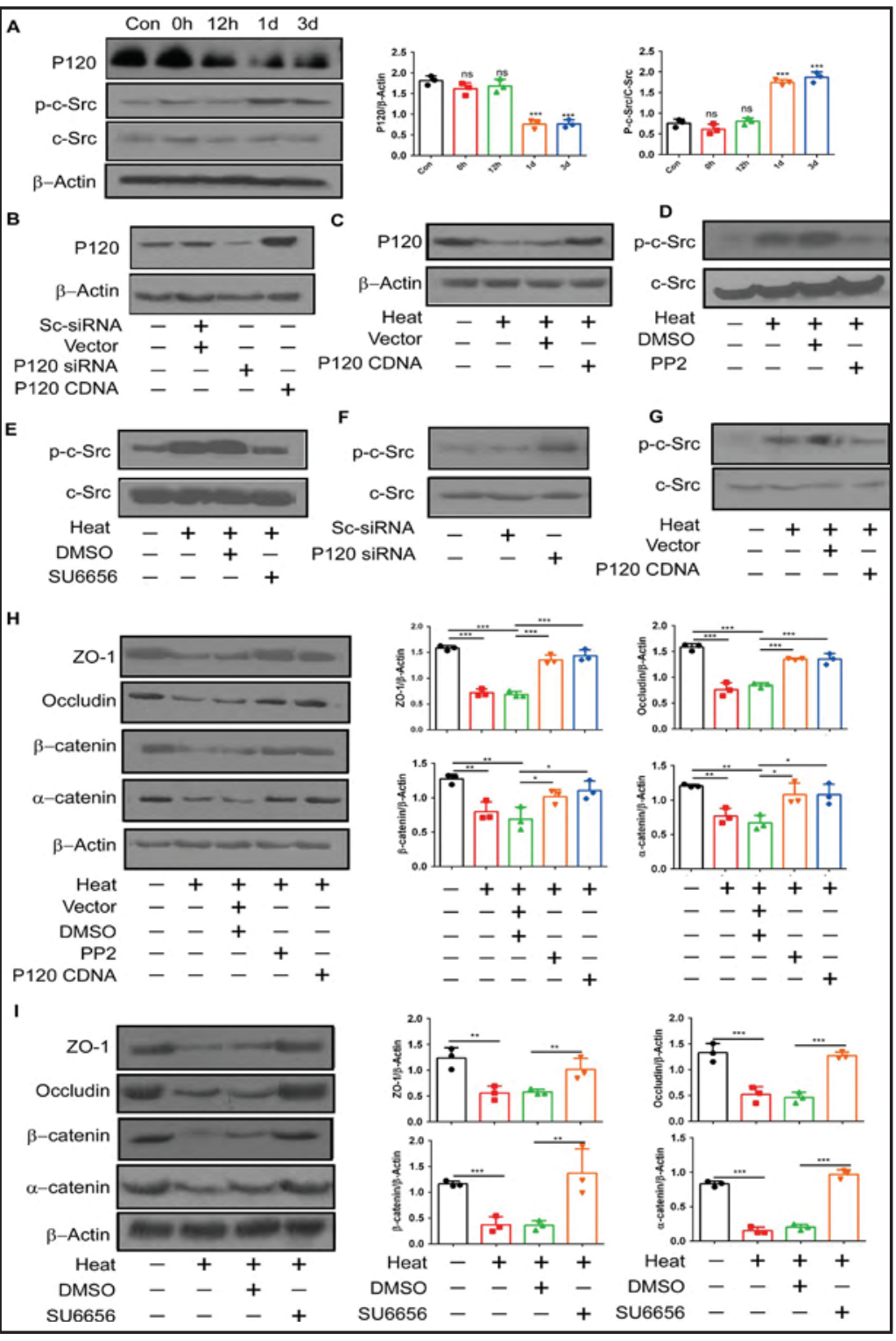
western blots of p-c-

Src and c-Srcp120ctn after p120ctn complementary DNA (cDNA) transfection in heat-treated cells. (H) Representative western blot and quantification data for the cell junction proteins ZO-1, occludin, $\beta$-catenin and $\alpha$-catenin after p120ctn siRNA, p120ctn cDNA and PP2 treatment. (I) Representative western blot and quantification data for the cell junction proteins ZO-1, occludin, $\beta$-catenin and $\alpha$-catenin after SU6656 treatment. ns, no significant difference, ${ }^{* * *} \mathrm{P}<0.0001$ vs. control.

Heat treatment decreased junction protein expression levels and increased paracellular permeability in vivo

Because heat can disturb junction protein integrity and increase cell permeability in vitro, we investigated the effect of heat on lung injury in vivo. The W/D weight ratio of lung tissue was used to quantify pulmonary edema. After thermal inhalation injury, the W/D weight ratios were increased on days 3 and 5 , which means that pulmonary edema occurred in the thermal inhalation injury model (Fig. 5A). As shown in Fig. 5B, compared with the sham group, the injury group had significantly lower mRNA expression levels of occludin, ZO-1, $\alpha$-catenin and $\beta$-catenin on days 2 and 5. Similarly, tight junction protein expression levels were the lowest on days 2 and 5 (Fig. 5C). 


\section{Cellular Physiology \\ Cell Physiol Biochem 2018;48:237-250 and Biochemistry

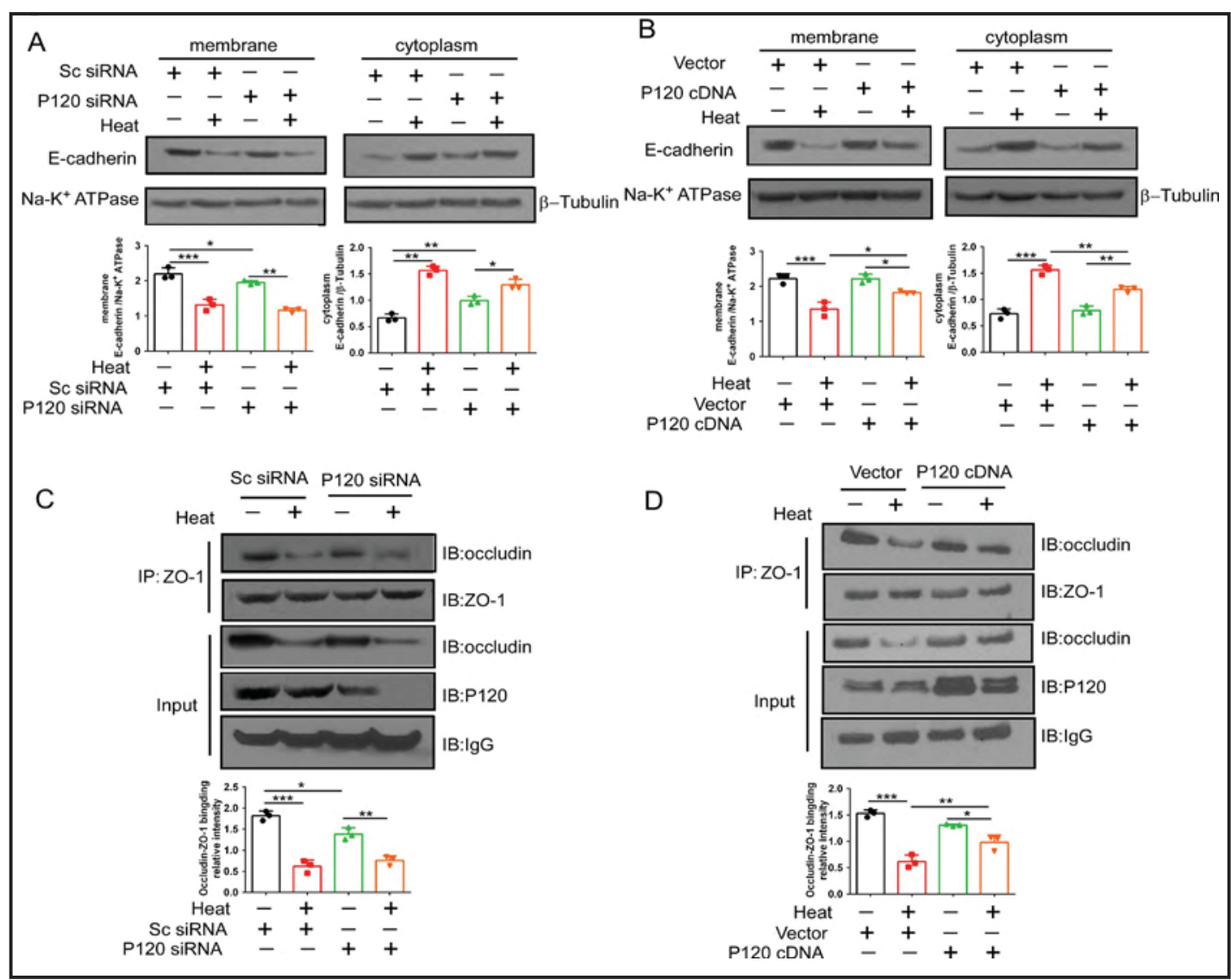

Fig. 4. Effect of p120ctn on E-cadherin distribution and junction binding in the heat treatment model. (A) Representative western blot and quantification data for E-cadherin in the cell membrane and cytoplasm after $\mathrm{p} 120 \mathrm{ctn}$ siRNA treatment. ns, no significant difference, $\mathrm{P}^{*}<0.05,{ }^{* *} \mathrm{P}<0.01$ and ${ }^{* * *} \mathrm{P}<0.0001$ vs. control $(n \geq 3)$. (B) Representative western blot and quantification data for E-cadherin in the cell membrane and cytoplasm after p120ctn cDNA treatment. ns, no significant difference, ${ }^{*} \mathrm{P}<0.05$, ${ }^{* *} \mathrm{P}<0.01$ and ${ }^{* * *} \mathrm{P}<0.0001$ vs. control $(\mathrm{n} \geq 3)$. (C) Binding between occludin and ZO-1 after p120ctn siRNA transfection was analyzed with Co-IP. ${ }^{*} \mathrm{P}<0.05$ and ${ }^{* * *} \mathrm{P}<0.001$ vs. control ( $\mathrm{n} \geq 3$ ). (D) Binding between occludin and $\mathrm{ZO}-1$ after $\mathrm{p} 120 \mathrm{cth}$ cDNA transfection was analyzed with Co-IP. $\mathrm{P}^{*}<0.05$ and ${ }^{* * *} \mathrm{P}<0.001$ vs. control $(\mathrm{n} \geq 3)$.

\section{Effects of p120ctn and c-Src on thermal inhalation lung injury}

Compared with the heat group, the p120ctn cDNA and PP2 groups had significantly lower W/D ratios (Fig. 6A). Furthermore, the expression levels of ZO-1, occludin, $\beta$-catenin and $\alpha$-catenin were higher in the P120CTN CDNA and PP2 treatment groups (Fig. 6C), which indicated that $\mathrm{p} 120 \mathrm{ctn} \mathrm{cDNA}$ and PP2 treatment may have protective effects on the junction protein degradation induced by thermal inhalation injury.

Epithelial barrier dysfunction in the lungs can induce inflammatory cell infiltration and lung edema and increase lung permeability. Lung pathological injury scores can indicate lung permeability [15]. The degree of lung pathological injury was scored based on the following variables: inflammatory cell infiltration, lung edema, hemorrhaging, hyaline membrane structure and atelectasis. As shown in Fig. 6D, lung pathological injury scores were three times higher than those of the control group. The histopathologic examination results showed increased numbers of inflammatory cells and red cells in the lungs from the thermal inhalation injury group. The epithelial integrity and lung permeability were significantly increased after heat treatment. Notably, all of these effects were markedly limited in the p120ctn cDNA and PP2 treatment groups (Fig. 6D). As shown in Fig. 6E and Fig. 6F, occludin and ZO-1 expression was detected mainly in the cytoplasmic membranes or peripheral cytoplasm of endothelial cells and the alveolar epithelium, and staining was more 


\section{Cellular Physiology \\ Cell Physiol Biochem 2018;48:237-250 and Biochemistry

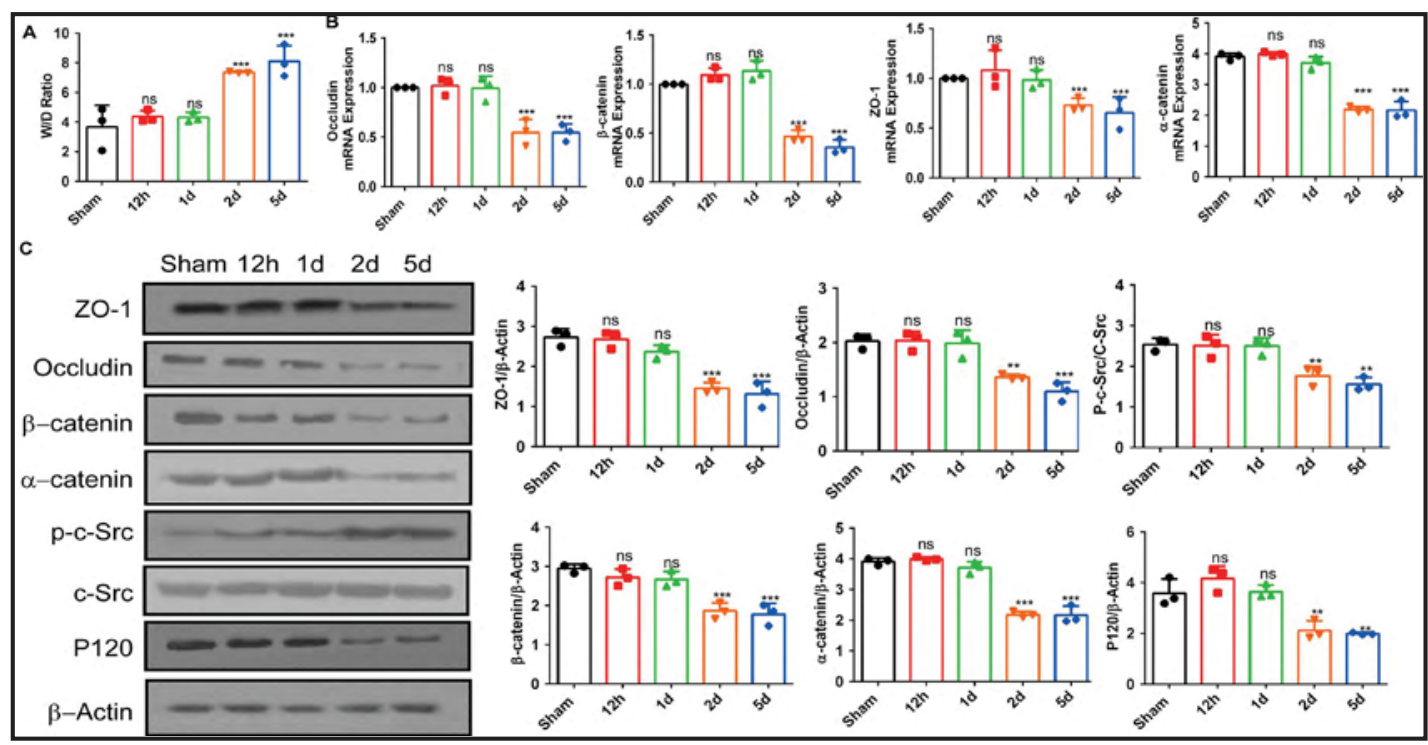

Fig. 5. Heat treatment disturbed junction protein expression and increased paracellular permeability in vivo. (A) The wet-to-dry (W/D) weight ratio was determined as detailed in the Materials and Methods section. The data shown are the means \pm SEM of at least three independent experiments $(\mathrm{n}=6$ in each group). ns, no significant difference, ${ }^{* *} \mathrm{P}<0.01$ and ${ }^{* * *} \mathrm{P}<0.0001$ vs. the sham group. (B) Real-time polymerase chain reaction and quantification data for the cell junction proteins ZO-1, occludin, $\beta$-catenin and $\alpha$-catenin. Relative mRNA levels were normalized to the $18 \mathrm{~s}$ ribosomal RNA levels. ns, no significant difference, ${ }^{* *} \mathrm{P}<0.01$ and ${ }^{* * *} \mathrm{P}<0.0001$ vs. the sham group $(\mathrm{n} \geq 3)$. (C) Representative western blots and quantification data for the cell junction proteins ZO-1, occludin, p120ctn, $\beta$-catenin, p-c-Src and $\alpha$-catenin. $\mathrm{P}^{*}<0.05,{ }^{* *} \mathrm{P}<0.01$ and ${ }^{* * *} \mathrm{P}<0.0001$ vs. the sham group.

intense at the junctions of adjacent cells. Compared with the heat treatment group, the PP2 and p120ctn cDNA treatment groups had significantly higher levels of occludin and ZO-1 expression.

\section{Discussion}

In the present study, we found that heat-induced c-Src activation led to p120ctn degradation, which reduced the disassociation and binding of junction proteins in vitro and in vivo. In a thermal inhalation injury model, heat treatment induced junction protein degradation and dissociation, resulting in increased alveolar membrane permeability and lung edema via c-Src kinase activation and p120ctn expression downregulation. This study is the first to elucidate the mechanism through which heat affects paracellular lung permeability and induces lung edema, and these findings may open new avenues for the therapy of thermal inhalation injury.

Several studies have shown that lung edema, acute lung injury and acute respiratory distress syndrome are caused by barrier malfunctions [18]. The disruption of intercellular junctions caused by heat is a major factor in epithelial permeability [19]. Accumulating evidence in animal and cell culture models indicates that the junctional malfunction of the lung barrier contributes significantly to deleterious outcomes. Moreover, heat has long been reported to induce intestinal barrier dysfunction through various pathways [20]. Consistent with these results, we found that in thermal inhalation injury, heat induces structural and cytosolic changes in alveolar epithelial cells and ultimately causes alveolar epithelial barrier dysfunction and pulmonary hyperpermeability.

In a conflagration, the temperature of the air surrounding the fire source is approximately $800^{\circ} \mathrm{C}[21,22]$. However, the temperature of the air in the bronchi is approximately $48.3^{\circ} \mathrm{C}$ 


\section{Cellular Physiology Cell Physiol Biochem 2018;48:237-250 \begin{tabular}{c|c|c|} 
DOI: 10.1159/000491723 & $\begin{array}{l}\text { O } 2018 \text { The Author(s). Published by S. Karger AG, Basel } \\
\text { www.karger.com/cpb }\end{array}$ \\
\hline
\end{tabular}}

Li et al.: Epithelial Barrier Dysfunction Occurs via C-Src Kinase and P120ctn Expression Regulation

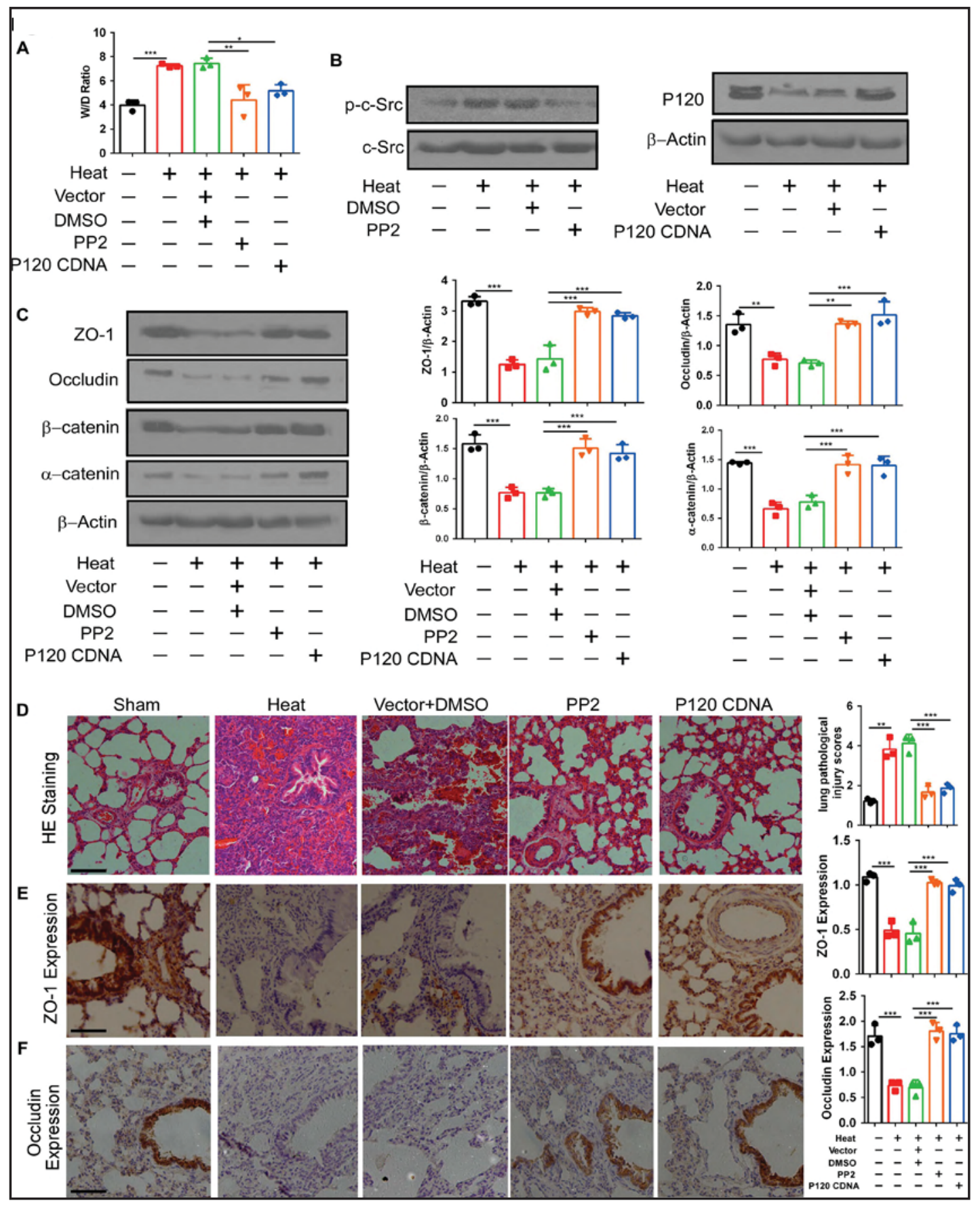

Fig. 6. Heat treatment disturbed junction protein expression and increased paracellular permeability in vivo. (A) The W/D weight ratios after PP2 and p120ctn cDNA treatment were determined as detailed in the Materials and Methods section. The data shown are the means \pm SEM of at least three independent experiments ( $\mathrm{n}=6$ in each group). ns, no significant difference, ${ }^{* *} \mathrm{P}<0.01$ and ${ }^{* * *} \mathrm{P}<0.0001$ vs. the sham group. (B) Western blot data for p-c-Src and p120ctn after PP2 and p120ctn cDNA treatment in vivo. (C) Representative western blot and quantification data for the cell junction proteins ZO-1, occludin, $\beta$-catenin and $\alpha$-catenin. $\mathrm{P}^{*}<0.05$, ${ }^{* *} \mathrm{P}<0.01$ and ${ }^{* * *} \mathrm{P}<0.0001$. (D) Hematoxylin and eosin staining showing lung permeability after inhalation injury. (E) Representative immunohistochemistry quantification data showing the localization and expression of ZO-1 in lung tissues after inhalation injury. (F) Representative immunohistochemistry quantification data showing the localization and expression of occludin in lung tissue after inhalation injury. 


\section{Cellular Physiology Cell Physiol Biochem 2018;48:237-250

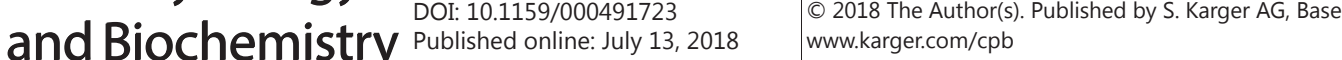 \\ Li et al.: Epithelial Barrier Dysfunction Occurs via C-Src Kinase and P120ctn Expression Regulation}

because more than $90 \%$ of the inhaled heat is released after passing through the upper airway. In the intestines, heat stress $\left(39-41^{\circ} \mathrm{C}\right)$ can disturb junction proteins and barrier function [23]. Accordingly, we created a heat damage model with cell exposure to $47^{\circ} \mathrm{C}$ for a short time to mimic the temperature at which inhalation injury occurs in burn patients.

In lung epithelial cells and the lung barrier, heat stress can induce lung intercellular junction degradation and increase lung permeability $[2,18,24]$. Some studies of intestinal cells have found that after heat treatment, junction protein expression levels increase initially and then decrease dramatically approximately $12 \mathrm{~h}$ after treatment. However, we observed no change in junction protein expression immediately after heat treatment; instead, we found a dramatic decrease on day 1 in the in vitro model and on day 2 in the in vivo model [25]. This difference may be attributed to differences in the cell lines used and the organs studied. Clinically, lung edema usually occurs on day 5 after thermal inhalation injury, which is consistent with our results.

Cell adherens junctions and tight junctions are key components in maintaining lung epithelial membrane integrity and permeability [26]. In particular, tight junctions are located on the outside of pulmonary capillary endothelial and epithelial cells and are composed of protein complexes containing claudins, ZO-1, junctional adhesion molecules (JAMs) and occludins; these junctions seal epithelial barriers to maintain pulmonary vascular permeability $[27,28]$. Claudin and JAM-1 were shown to be associated with ZO-1 through both non-PDZ and PDZ domains [29]. Although more proteins are present, the major types are ZO- 1 and the occludins, which can bind to the actin component of the cytoskeleton. Thus, occludin and ZO-1 are always used as the major makers for tight junctions [30]. Mark et al. found that the downregulation or redistribution of occludin and ZO-1 in intestinal epithelial cells impaired the function of the intestinal epithelial barrier, causing malabsorption and diarrhea [26, 31]. Adherens junctions depend on the associations of p120ctn, E-cadherin, $\beta$-catenin and $\alpha$-catenin, which mediate the transduction of stress signals to vascular endothelial cells [32]. $\beta$-catenin acts by anchoring the actin cytoskeleton to the junctions. $\alpha$-catenin participates in the formation and stabilization of adherens junctions by binding to $\beta$-catenin-cadherin complexes in the cell [33]. Additional catenins, such as $\gamma$-catenin and $\delta$-catenin, have been identified. Most studies investigating catenin actions focus on $\alpha$-catenin and $\beta$-catenin [34]. However, it is unclear whether the regulation of occludin and ZO-1 is related to lung tissue permeability in thermal inhalation injury. Therefore, we measured ZO1 , occludin, $\beta$-catenin and $\alpha$ - catenin expression after heat treatment. Our data showed that occludin and ZO-1 expression levels were decreased in thermal inhalation injury, reaching their lowest levels 2 days after injury; these decreases induced lung tissue permeability. These changes correlated with changes in lung tissue permeability, which suggests that structural abnormalities in junction proteins were responsible for the lung damage.

p120ctn plays a key role in lung injury induced by mechanical ventilation [12, 35]. It is expressed at high levels in pulmonary vascular endothelial cells, lung epithelial cells, fibroblasts, cardiomyocytes and muscle cells; in these cell types, it co-functions with E-cadherin to maintain intercellular adhesion junction stability. E-cadherin facilitates the assembly of specialized cellular junctions required to organize epithelial cells into a functional monolayer. Changes in the structure and function of the cells disturb alveolar membrane integrity and decrease cell junction protein expression, further increasing pulmonary vascular permeability [36]. The results of our study showed that heat treatment disrupted the association of p120ctn and E-cadherin. We also found that intercellular junction endocytosis and degradation were enhanced after p120ctn depletion both in vitro and in vivo. Moreover, p120ctn knockdown significantly downregulated the association of occludin and ZO-1 under normal conditions. After heat treatment, p120ctn overexpression reversed the heat-induced downregulation of occludin and ZO-1 association. In vivo, p120ctn overexpression decreased the lung $\mathrm{W} / \mathrm{D}$ weight ratio and reversed the intercellular junction dysfunction caused by heat. Furthermore, compared with the scrambled siRNA group, the P120CTN siRNA group showed decreased E-cadherin expression levels in the cell membrane after heat treatment, and occludin and ZO-1 association was downregulated (see Fig. 4A 


\section{Cellular Physiology Cell Physiol Biochem 2018;48:237-250 \begin{tabular}{c|c} 
DOI: $10.1159 / 000491723$ \\
and Biochemistry Published online: July 13, 2018 & $\begin{array}{l}\text { O } 2018 \text { The Author(s). Published by S. Karger AG, Basel } \\
\text { www.karger.com/cpb }\end{array}$ \\
\cline { 2 - 2 } &
\end{tabular} \\ Li et al.: Epithelial Barrier Dysfunction Occurs via C-Src Kinase and P120ctn Expression Regulation}

and 4c); these results indicate that other pathways also regulate intercellular junctions in thermal inhalation injury.

Src kinase is a critical protein for cell signal transmission, and it participates in cell adhesion, growth, migration, differentiation and necrosis [11,37]. Moreover, Src plays a key role in regulating lung tissue inflammation. Mechanical stretch stimulation reportedly activates Src kinase and causes cell attachment protein degradation. PP2, a Src kinasespecific inhibitor, effectively blocks the mechanical stretch-induced degradation of cell junction proteins, further reducing lung injury [38]. In our study, after heat treatment, Src kinase activity was significantly increased, resulting in cell junction protein degradation and accelerated lung edema development. The c-Src inhibitor PP2 effectively limited cell junction protein degradation, which indicates that heat caused the junction protein degradation to induce lung injury by activating Src kinase. Consistent with previous studies, our results also showed that p120ctn degradation was associated with c-Src activation [27, 28]. Moreover, PP2 reversed the heat-induced degradation of p120ctn in vitro and in vivo. These results indicate that c-Src is a critical and upstream signaling protein in thermal inhalation injury models.

This study has several limitations, and further research is needed to clarify our findings. First, we used an immortal cell line for the in vitro studies. Because it is unethical to perform a study of this nature in vivo in humans, a new technique is required to improve the applicability of this research to human patients. Second, Ley and Arfors developed a protocol in which intravenous FITC-dextran was used with fluorescence microscopy to monitor changes in microvascular leakage in intact tissues. This technique could be used for further studies in vivo [39]. Third, temperature is likely a core factor in compromised lung barrier function, which leads to lung edema. However, the mechanism through which heat affects lung barrier permeability is complicated and includes inflammatory cytokine activity as well as endotoxemia and the innate immune dysfunction of the mitochondria or endoplasmic reticulum [5, 40], which may become a network pathway between cells. Additional research is needed to elucidate this mechanism more clearly. Fourth, a microarray experiment was devised to monitor changes in gene expression in the injured skin from 45 burn patients compared to normal skin from 15 healthy patients. According to a global gene microarray analysis of cutaneous burn wounds, there was a dataset of 1136 upregulated genes and 1003 downregulated genes during the 1-17-day period after burn trauma [41]. Jonathan et al. also present a global characterization of genes and proteins related to the lung response to heat stress in a rat model, and they found 78 differentially expressed transcripts in the lung, including transcripts related to the unfolded protein response, inflammation, and apoptosis [42]. A global gene microarray analysis was also needed for the epithelia after the heat treatment.

In summary, our findings indicate that heat treatment induces lung tissue permeability and lung edema by activating c-Src kinase, which in turn downregulates p120ctn expression. This study may provide potential targets for the development of new drugs that can protect lung function from further damage in thermal injury.

\section{Acknowledgements}

This work was supported by the National Natural Science Foundation of China (NSFC: 81770028), the Natural Science Foundation of Guangdong (2016A030310087), the Project of Shenzhen Basic Research Plan (JCYJ20160422165836057 and JCYJ20170307095633450), the China Postdoctoral Science Foundation (2016M592602) and clinical research of the Health and Family Planning Commission of Shenzhen Municipality (SZLY2017024).

\section{Disclosure Statement}

The authors of this article declare no conflict of interests. 


\section{Cellular Physiology Cell Physiol Biochem 2018;48:237-250 \begin{tabular}{l|l|l} 
DOI: 10.1159/000491723 & (0) 2018 The Author(s). Published by S. Karger AG, Basel
\end{tabular} \begin{tabular}{l|l} 
Published online: July 13, 2018 www.karger.com/cpb \\
\hline
\end{tabular} \\ Li et al.: Epithelial Barrier Dysfunction Occurs via C-Src Kinase and P120ctn Expression Regulation}

\section{References}

1 Flouris AD, Kenny GP: Heat remains unaccounted for in thermal physiology and climate change research. F1000Res 2017;6:221.

2 Vo E, Kurmis R, Campbell J, Greenwood J: Risk factors for and characteristics of dysphagia development in thermal burn injury and/or inhalation injury patients: a systematic review protocol. JBI Database System Rev Implement Rep 2016;14:31-43.

-3 Wohlsein P, Peters M, Schulze C, Baumgartner W: Thermal Injuries in Veterinary Forensic Pathology. Vet Pathol 2016;53:1001-1017.

-4 Zhao Y, Zhang M, Xiong RP, Chen XY, Li P, Ning YL, Yang N, Peng Y, Zhou YG: Somatostatin Reduces the Acute Lung Injury of Mice via Increasing the Affinity of Glucocorticoid Receptor. Cell Physiol Biochem 2016;38:1354-1364.

5 Dries DJ, Endorf FW: Inhalation injury: epidemiology, pathology, treatment strategies. Scand J Trauma Resusc Emerg Med 2013;21:31.

-6 Fei ZW, Young VR, Lu XM, Rhodes AB, Tompkins RG, Fischman AJ, Yu YM: Burn injury differentially alters whole-blood and organ glutathione synthesis rates: An experimental model. Burns Trauma 2013;1:87-94.

7 Toon MH, Maybauer MO, Greenwood JE, Maybauer DM, Fraser JF: Management of acute smoke inhalation injury. Crit Care Resusc 2010;12:53-61.

8 Gulino-Debrac D: Mechanotransduction at the basis of endothelial barrier function. Tissue Barriers 2013;1:e24180.

9 Birukov KG, Zebda N, Birukova AA: Barrier enhancing signals in pulmonary edema. Compr Physiol 2013;3:429-484.

10 Coopman P, Djiane A: Adherens Junction and E-Cadherin complex regulation by epithelial polarity. Cell Mol Life Sci 2016;73:3535-3553.

-11 Huang Y, He Q: Inhibition of c-Src protects paraquat induced microvascular endothelial injury by modulating caveolin-1 phosphorylation and caveolae mediated transcellular permeability. Environ Toxicol Pharmacol 2017;52:62-68.

12 Zhao T, Zhao H, Li G, Zheng S, Liu M, Gu C, Wang Y: Role of the PKCalpha-c-Src tyrosine kinase pathway in the mediation of p120-catenin degradation in ventilator-induced lung injury. Respirology 2016;21:14041410.

13 Forgati M, Kandalski PK, Herrerias T, Zaleski T, Machado C, Souza MR, Donatti L: Effects of heat stress on the renal and branchial carbohydrate metabolism and antioxidant system of Antarctic fish. J Comp Physiol B 2017;10.1007/s00360-017-1088-3

14 Dong ZW, Chen J, Ruan YC, Zhou T, Chen Y, Chen Y, Tsang LL, Chan HC, Peng YZ: CFTR-regulated MAPK/NFkappaB signaling in pulmonary inflammation in thermal inhalation injury. Sci Rep 2015;5:15946.

15 Sun CZ, Shen H, He XW, Bao L, Song Y, Zhang Z, Qin HD: Effect of dobutamine on lung aquaporin 5 in endotoxine shock-induced acute lung injury rabbit. J Thorac Dis 2015;7:1467-1477.

16 Gohy ST, Hupin C, Fregimilicka C, Detry BR, Bouzin C, Gaide Chevronay H, Lecocq M, Weynand B, Ladjemi MZ, Pierreux CE, Birembaut P, Polette M, Pilette C: Imprinting of the COPD airway epithelium for dedifferentiation and mesenchymal transition. Eur Respir J 2015;45:1258-1272.

-17 Fernandez-Gil B, Moneim AE, Ortiz F, Shen YQ, Soto-Mercado V, Mendivil-Perez M, Guerra-Librero A, AcunaCastroviejo D, Molina-Navarro MM, Garcia-Verdugo JM, Sayed RK, Florido J, Luna JD, Lopez LC, Escames G: Melatonin protects rats from radiotherapy-induced small intestine toxicity. PLoS One 2017;12:e0174474.

18 Baxter PJ, Jenkins S, Seswandhana R, Komorowski JC, Dunn K, Purser D, Voight B, Shelley I: Human survival in volcanic eruptions: Thermal injuries in pyroclastic surges, their causes, prognosis and emergency management. Burns 2017;10.1016/j.burns.2017.01.025

19 Kumar AB, Andrews W, Shi Y, Shotwell MS, Dennis S, Wanderer J, Summitt B: Fluid resuscitation mediates the association between inhalational burn injury and acute kidney injury in the major burn population. J Crit Care 2017;38:62-67.

-20 O’Donnell JJ, 3rd, Birukova AA, Beyer EC, Birukov KG: Gap junction protein connexin43 exacerbates lung vascular permeability. PLoS One 2014;9:e100931.

21 Bailer AJ, Bena JF, Stayner LT, Halperin WE, Park RM: External cause-specific summaries of occupational fatal injuries. Part II: an analysis of years of potential life lost. Am J Ind Med 2003;43:251-261. 


\section{Cellular Physiology Cell Physiol Biochem 2018;48:237-250 \begin{tabular}{l|l} 
and Biochemistry Published online: July 13, 2018 & $\begin{array}{l}\text { D 2018 The Author(s). Published by S. Karger AG, Basel } \\
\text { www.karger.com/cpb }\end{array}$ \\
\cline { 2 - 3 }
\end{tabular} \\ Li et al.: Epithelial Barrier Dysfunction Occurs via C-Src Kinase and P120ctn Expression Regulation}

22 Baldwin DO: Public health services and limited prospects: epidemic and conflagration in Cobalt. Ont Hist 1983;75:374-402.

-23 Ozcinar E, Cakici M, Korun O, Han U, Kiziltepe U: Comparison of heat induced damage at the saphenofemoral junction after ablation with 1, $470 \mathrm{~nm}$ laser or radiofrequency. Vasa 2017;46:101-107.

24 He J, Xiang Z, Xiao J, Xiao H, Liu L: [The poor chemotherapeutic efficacy in lung adenocarcinoma overexpressing c-Src and nucleophosmin/B23(NPM1)]. Xi Bao Yu Fen Zi Mian Yi Xue Za Zhi 2016;32:13781381.

25 Yi G, Li L, Luo M, He X, Zou Z, Gu Z, Su L: Heat stress induces intestinal injury through lysosome- and mitochondria-dependent pathway in vivo and in vitro. Oncotarget 2017;10.18632/oncotarget.16580

-26 Mark KS, Davis TP: Cerebral microvascular changes in permeability and tight junctions induced by hypoxiareoxygenation. Am J Physiol Heart Circ Physiol 2002;282:H1485-1494.

27 Cho RL, Yang CC, Lee IT, Lin CC, Chi PL, Hsiao LD, Yang CM: Lipopolysaccharide induces ICAM-1 expression via a c-Src/NADPH oxidase/ROS-dependent NF-kappaB pathway in human pulmonary alveolar epithelial cells. Am J Physiol Lung Cell Mol Physiol 2016;310:L639-657.

28 Royhman D, Patel M, Runa MJ, Wimmer MA, Jacobs JJ, Hallab NJ, Mathew MT: Fretting-corrosion behavior in hip implant modular junctions: The influence of friction energy and pH variation. Biol Open 2016;62:570587.

29 Arcangeli ML, Frontera V, Aurrand-Lions M: Function of junctional adhesion molecules (JAMs) in leukocyte migration and homeostasis. Arch Immunol Ther Exp (Warsz) 2013;61:15-23.

30 Li X, Liu X, Yu S: Psychological Stress-Derived Prolactin Modulates Occludin Expression in Vaginal Epithelial Cells to Compromise Barrier Function. Cell Physiol Biochem 2015;37:153-161.

-31 Abbruscato TJ, Lopez SP, Mark KS, Hawkins BT, Davis TP: Nicotine and cotinine modulate cerebral microvascular permeability and protein expression of ZO-1 through nicotinic acetylcholine receptors expressed on brain endothelial cells. J Pharm Sci 2002;91:2525-2538.

-32 Wang R, Chen YS, Dashwood WM, Li Q, Lohr CV, Fischer K, Ho E, Williams DE, Dashwood RH: Divergent roles of p120-catenin isoforms linked to altered cell viability, proliferation, and invasiveness in carcinogeninduced rat skin tumors. Mol Carcinog 2017;10.1002/mc.22630

-33 Kang H, Bang I, Jin KS, Lee B, Lee J, Shao X, Heier JA, Kwiatkowski AV, Nelson WJ, Hardin J, Weis WI, Choi HJ: Structural and functional characterization of Caenorhabditis elegans alpha-catenin reveals constitutive binding to beta-catenin and F-actin. J Biol Chem 2017;292:7077-7086.

-34 Chen CY, Zhang M, Wang SS, Lu B, Wang X: [Changes of adherens junctions between the epithelial cells of intestinal mucosal barrier in irritable bowel syndrome]. Zhonghua Yi Xue Za Zhi 2017;97:1065-1070.

-35 Dai CY, Dai GF, Sun Y, Wang YL: Loss of p120 catenin aggravates alveolar edema of ventilation induced lung injury. Chin Med J (Engl) 2013;126:2918-2922.

-36 Tanaka S, Chen-Yoshikawa TF, Miyamoto E, Takahashi M, Ohata K, Kondo T, Hijiya K, Motoyama H, Aoyama A, Date H: Vascular Endothelial-Cadherin Expression After Reperfusion Correlates With Lung Injury in Rat Lung Transplantation. Ann Thorac Surg 2016;101:2161-2167.

-37 Hyun SW, Liu A, Liu Z, Lillehoj EP, Madri JA, Reynolds AB, Goldblum SE: As human lung microvascular endothelia achieve confluence, src family kinases are activated, and tyrosine-phosphorylated p120 catenin physically couples NEU1 sialidase to CD31. Cell Signal 2017;35:1-15.

-38 Tian Y, Gawlak G, O’Donnell JJ, 3rd, Birukova AA, Birukov KG: Activation of Vascular Endothelial Growth Factor (VEGF) Receptor 2 Mediates Endothelial Permeability Caused by Cyclic Stretch. J Biol Chem 2016;291:10032-10045.

-39 Zhang J, Jin H, Liu H, Lv S, Wang B, Wang R, Liu H, Ding M, Yang Y, Li L, Zhang J, Fu S, Xie D, Wu M, Zhou W, Qian Q: MiRNA-99a directly regulates AGO2 through translational repression in hepatocellular carcinoma. Oncogenesis 2014;3:e97.

40 Buettner K: Effects of extreme heat on man; protection of man against conflagration heat. J Am Med Assoc 1950;144:732-738.

41 Greco JA, 3rd, Pollins AC, Boone BE, Levy SE, Nanney LB: A microarray analysis of temporal gene expression profiles in thermally injured human skin. Burns 2010;36:192-204.

-42 Stallings JD, Ippolito DL, Rakesh V, Baer CE, Dennis WE, Helwig BG, Jackson DA, Leon LR, Lewis JA, Reifman J: Patterns of gene expression associated with recovery and injury in heat-stressed rats. BMC Genomics 2014; $15: 1058$. 